\title{
Revisión, diseño y construcción de un instrumento de medición para evaluar las competencias laborales del personal farmacéutico ambulatorio
}

\section{Review, design, and construction of a measuring instrument to assess the labor competencies of outpatient pharmacy staff}

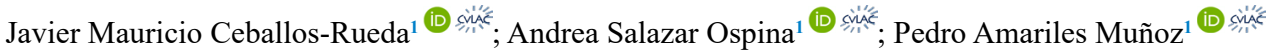

Forma de citar: Ceballos Rueda JM, Salazar Ospina A, Amariles Muñoz P. Revisión, diseño y construcción de un instrumento de medición para evaluar las competencias laborales del personal farmacéutico ambulatorio. Salud UIS. 2021; e21027. doi: https://doi.org/10.18273/saluduis.53.e:21027 (c) (i)

\section{Resumen}

Introducción: la medición y evaluación de las competencias laborales, en el personal farmacéutico ambulatorio, debe combinar aspectos relacionados con los conocimientos (que sabe), habilidades (como lo hace) y actitudes (de que modo lo hace). Objetivo: identificar y seleccionar las directrices y recomendaciones sobre las competencias laborales que requiere el personal farmacéutico ambulatorio, y construir un instrumento para su medición. Métodos: se realizó una revisión estructurada en dos fases: a) se revisaron y seleccionaron las funciones/dominios, los criterios de desempeño (habilidades y actitudes), los conocimientos, los marcos de competencias establecidas a nivel internacional, y los instrumentos de medición reportados en la literatura; y b) se identificaron las competencias laborales necesarias de acuerdo con la normatividad colombiana y con los procesos de los Establecimientos Farmacéuticos ambulatorios (droguerías y farmacias-droguerías). Finalmente, se construyó el instrumento de medición por un panel de expertos. Resultados: se identificaron las competencias laborales establecidas por las organizaciones de regulación internacional. Se revisaron 993 artículos que reportaban la medición y evaluación de las competencias, seleccionándose y analizándose 9 referencias. Se analizaron los marcos de competencias de diferentes países. Se analizaron las regulaciones en Colombia sobre el tema. El instrumento construido contiene 101 competencias distribuidas en los siguientes componentes: a) suministro de cuidados al paciente; b) personales; c) solución de problemas; y d) gestión y organización. Conclusión: directrices y recomendaciones internacionales, además, la normatividad colombiana para identificar las competencias laborales que requiere el personal farmacéutico para el correcto funcionamiento de los Establecimientos Farmacéuticos ambulatorios, y partir de esto, se construye un instrumento de medición para evaluarlas.

Palabras clave: Competencias Laborales; Personal Farmacéutico; Conocimientos; Habilidades y Actitudes en Salud; Farmacias; Establecimientos Farmacéuticos.

1. Universidad de Antioquia. Medellín, Colombia.

Correspondencia: Mauricio Ceballos. Dirección: Calle 67 53-108, Medellín. Teléfono: +57 (4) 2198332. Correo electrónico: javier.ceballos@udea.edu.co 


\begin{abstract}
Introduction: The measurement and evaluation of labor competencies, in outpatient pharmacy staff, must combine aspects related to knowledge (who knows), skills (as it does) and attitudes (in what way it does). Objective: Identify and select the guidelines and recommendations on labor competencies required by outpatient pharmacy staff and build an instrument for their measurement. Methodology: A structured review was carried out in two phases: a) the functions/ domains, performance criteria (skills and attitudes), knowledge, competency frameworks established internationally, and measurement instruments reported were reviewed and selected in the literature; and b) the necessary labor competencies were identified in accordance with Colombian regulations and with the processes of the outpatient Pharmaceutical Establishments (drugstores and pharmacies-drugstores). Finally, the measuring instrument was built by a panel of experts. Results: Labor competencies established by international regulation organizations. were identified. A total of 993 articles were reviewed that reported the measurement and evaluation of competencies, selecting and analyzing 9 references. Competency frameworks from different countries were analyzed. The regulations in Colombia on the subject were analyzed. The constructed instrument contains 101 competencies distributed in the following components: a) provision of patient care; b) personal; c) troubleshooting; and d) management and organization. Conclusion: The international guidelines and recommendations were reviewed and analyzed, in addition, the Colombian regulations to identify the labor competencies required by the pharmacy staff for the correct functioning of the outpatient Pharmaceutical Establishments, and from this, an instrument was built of measurement to evaluate them.
\end{abstract}

Keywords: Labor Competencies; Pharmacy Staff; Knowledge; Skills and Attitudes in Health; Pharmacies; Pharmaceutical Establishments.

\section{Introducción}

La Organización Mundial de la Salud (OMS), la Organización Panamericana de la Salud (OPS) y la Federación Internacional Farmacéutica (FIP) definen las competencias laborales "como los conocimientos, habilidades y actitudes requeridos por el conjunto de los recursos humanos de salud en los diferentes ámbitos de práctica, para solucionar los problemas de salud de manera eficiente y efectiva ${ }^{\text {"1,2. }}$. Una competencia laboral es un comportamiento que reúne diferentes elementos, criterios de desempeño y conocimientos que determinan el logro, dominio o cumplimiento de esta en un ambiente real de trabajo, que se pueden medir y evaluar a partir de la combinación de técnicas de investigación cualitativas y cuantitativas, como la observación y el uso de instrumentos de medición, respectivamente. Su medición y evaluación debe realizarse siempre de manera conjunta, mediante la combinación de aspectos relacionados con los conocimientos (qué sabe), habilidades (cómo lo hace) y actitudes (de qué modo lo hace).

Por otro lado, la normatividad colombiana define a las droguerías y las farmacias-droguerías como Establecimientos Farmacéuticos ambulatorios (minoristas) ${ }^{3}$, y es permitido que una persona labore dentro de estos establecimientos con alguno de los siguientes niveles de formación: profesional, tecnológico o técnico/auxiliar. Aunque los perfiles, áreas de formación y componentes fundamentales de cada uno se han establecido en la normatividad ${ }^{3-5}$, solo para el técnico o auxiliar de Servicios Farmacéuticos existe una norma de competencias laborales específica. Además, está el perfil laboral de expendedor de medicamentos (denominación dada por el Ministerio de Salud a aquellas personas con experiencia en el campo pero que no tienen formación universitaria profesional o tecnológica, también es denominado director de droguerías) ${ }^{3}$. Sobre este último perfil no hay un referente normativo que establezca las competencias laborales que debe cumplir. Debido a esta compleja combinación de perfiles laborales para el desempeño en estos establecimientos, es necesario asumir y utilizar el término "personal farmacéutico" para toda persona que labora en un Establecimiento Farmacéutico minorista, y cuyo nivel educativo puede ser profesional, tecnológico, técnico/auxiliar o empírico con experiencia. Además, se ha comprobado que una gran proporción de estos establecimientos, se encuentran dirigidos por personal que no cuentan con soportes de capacitación y educación continua ${ }^{6,7}$.

En Colombia no se dispone de un instrumento de medición para evaluar las competencias laborales para el personal farmacéutico ambulatorio. Por ello, es necesario revisar y construir un instrumento de medición que pueda satisfacer esta necesidad, con el fin de tener un referente para la generación de nuevos estudios e intervenciones enfocadas en esta área. En este sentido, los objetivos de este estudio fueron: 1) identificar las 
directrices y recomendaciones internacionales, además de la normatividad colombiana, para establecer y agrupar las competencias laborales que requiere el personal farmacéutico ambulatorio para el correcto funcionamiento de los Establecimientos Farmacéuticos ambulatorios y, con dicho soporte, 2) construir un instrumento de medición para evaluar las competencias laborales en este personal de salud.

\section{Metodología}

\section{Identificación de las competencias laborales y sus componentes}

a. Revisión, agrupación y selección de las competencias laborales establecidas a nivel nacional e internacional

Se realizó una búsqueda de los documentos de referencia de la OMS, OPS y FIP enfocadas en las directrices $\mathrm{y}$ recomendaciones relacionadas con el funcionamiento adecuado de las farmacias y los servicios farmacéuticos a nivel comunitario (ambulatorio), con el objetivo de revisar y seleccionar las funciones, tareas, actividades, elementos y criterios de desempeño de las competencias. Adicionalmente, se realizó una búsqueda libre para identificar asociaciones, agremiaciones y grupos de trabajo que se enfocaran en la medición, validación y/o caracterización de competencias laborales en el personal farmacéutico con el fin de identificar los marcos de competencias.

Se revisó la normatividad vigente en el contexto del sistema de salud colombiano, asociada con las competencias laborales del personal farmacéutico y, con los hallazgos de esta revisión, se agruparon las competencias, acorde con los procesos propios de las droguerías y las farmacias-droguerías. Para esta agrupación, se tuvo en cuenta los procesos y subprocesos descritos en del Decreto 780 de 2016 del Ministerio de Salud y Protección Social, el cual, regula las actividades y/o procesos propios y establece los objetivos de estos establecimientos. Finalmente, para la selección de las competencias laborales, se consideraron los siguientes criterios: 1) que las competencias estuvieran clasificadas dentro de los procesos generales y especiales de las droguerías y las farmacias-droguerías; 2) que los criterios de desempeño se enfocaran en el ámbito ambulatorio; y 3) que los conocimientos necesarios tuvieran relación con aspectos técnicos, administrativos y/o asistenciales del quehacer farmacéutico; por ello, se descartaron los conocimientos de las áreas básicas como anatomía, fisiología, primeros auxilios, matemáticas, contabilidad, informática e inglés.

\section{b. Revisión de instrumentos de medición de competencias laborales}

Se realizó una revisión estructurada en PubMed/ Medline de artículos publicados en inglés o español, con acceso a texto completo entre 2008 y 2018, sobre la medición y evaluación de competencias laborales en el personal farmacéutico ambulatorio. La búsqueda se realizó con los siguientes términos $\mathrm{MeSH} / \mathrm{DeCS}$ y operadores booleanos: "Professional Competence" OR "Health Knowledge, Attitudes, Practice" AND "Pharmacists". Los criterios de inclusión fueron: 1) contener en el título o resumen información sobre evaluación conjunta de competencias laborales; y 2) que el instrumento evaluara de forma global todas las competencias laborales. Por su parte, los criterios de exclusión fueron: 1) artículos sin ninguna relación con los objetivos de la búsqueda y de la revisión; y 2) artículos con información limitada para revisar y analizar el instrumento de medición. Los artículos incluidos fueron seleccionados por dos investigadores de forma pareada. Para ello, se revisaron los títulos y resúmenes de todos los artículos identificados para decidir su elegibilidad. Los artículos seleccionados se analizaron conjuntamente y, por consenso, se definió su inclusión o no. Se diseñó un formato en Excel 2016 para Windows ${ }^{\circledR}$ para consignar $\mathrm{y}$ tabular los datos de los estudios.

\section{Construcción del instrumento de medición}

Para la construcción del instrumento de medición se tuvieron en cuentas los dominios (funciones), competencias, elementos, criterios de desempeño y conocimientos identificados en las revisiones de la normatividad colombiana, en los marcos de competencias laborales de las OMS/OPS/FIP, y de los grupos de trabajo en este campo a nivel internacional, además, de los instrumentos de medición de las referencias analizadas en la revisión estructurada. El instrumento de medición se construyó de acuerdo con el contexto del sistema de salud colombiano, y teniendo en cuenta los detalles del proceso metodológico recomendado para estos casos, en el diseño de preguntas, la evaluación por expertos y la prueba piloto ${ }^{8}$.

\section{Resultados}

\section{Identificación de las competencias laborales y sus componentes}

a. Revisión, agrupación y selección de las competencias laborales establecidas a nivel nacional e internacional

Como fuentes de revisión se utilizaron los documentos de referencia internacional de la OMS/OPS/FIP en Buenas Prácticas en Farmacia (BPF) ${ }^{9}$, Servicios 
Farmacéuticos basados en Atención Primaria en Salud (APS) y las Buenas Prácticas en Farmaciaª y las competencias laborales en farmacéuticos ${ }^{10}$. Además, se revisaron diferentes marcos de competencias laborales para el desempeño asistencial del farmacéutico, que son implementadas y evaluadas en distintos países, como Inglaterra, Irlanda y Australia. Los dominios y competencias se clasificaron de acuerdo con la clasificación de la FIP/OMS. Los hallazgos se resumen en la Tabla 1.

Tabla 1. Funciones y dominios de los marcos de competencias laborales a nivel internacional de acuerdo con la FIP/OMS.

\begin{tabular}{|c|c|c|c|c|}
\hline \multirow{2}{*}{ Dominios } & \multirow{2}{*}{ Competencias } & \multicolumn{3}{|c|}{ Marcos de competencias } \\
\hline & & Inglaterra & Irlanda & Australia \\
\hline $\begin{array}{l}\text { Salud pública (políticas públicas, } \\
\text { organización y gestión de sistemas } \\
\text { y servicios farmacéuticos) }\end{array}$ & Gestión y organización & $\mathrm{x}$ & $\mathrm{x}$ & $\mathrm{x}$ \\
\hline Vinculadas al medicamento & Gestión y suministro de medicamentos & $\mathrm{x}$ & $\mathrm{x}$ & $\mathrm{x}$ \\
\hline Vinculadas al paciente & Cuidados y seguridad del paciente & $\mathrm{x}$ & $\mathrm{x}$ & $\mathrm{x}$ \\
\hline \multirow{2}{*}{ Profesionales y personales } & $\begin{array}{l}\text { Habilidades personales } \\
\text { (ética, comunicación, colaboración) }\end{array}$ & $\mathrm{x}$ & $\mathrm{x}$ & $\mathrm{x}$ \\
\hline & $\begin{array}{l}\text { Práctica y desarrollo profesional } \\
\text { (liderazgo, educación, investigación) }\end{array}$ & $\mathrm{x}$ & $\mathrm{x}$ & $\mathrm{x}$ \\
\hline
\end{tabular}

Posteriormente, se revisó la normatividad que regula las competencias laborales del personal farmacéutico en Colombia. Considerando que: 1) una gran proporción de Establecimientos Farmacéuticos ambulatorios, se encuentran dirigidos (amparados) por personal sin formación universitaria profesional o tecnológica (empírico autorizado por el ente de vigilancia y control para el ejercicio de la farmacia -expendedores de medicamentos o director de droguerías-); y 2) la ausencia de un referente normativo que establezca las competencias que debe tener este personal empírico; se estableció que las competencias laborales y sus componentes descritos en la normatividad, específicamente para los Auxiliares de Servicios Farmacéuticos, fueran las mínimas necesarias para la construcción del instrumento de medición. En este sentido, se identificaron 8 competencias laborales,
21 elementos, 181 criterios de desempeño, y 215 conocimientos y comprensiones.

Para la agrupación de las competencias laborales, se analizaron los criterios de desempeño identificados en el paso anterior y fueron distribuidos de acuerdo con el Decreto 780 de 2016, en: a) Procesos generales, y b) Procesos especiales y, posteriormente, fueron asignados en los diferentes subprocesos descritos en la misma norma. Esta agrupación fue seguida de la selección de $105(58 \%)$ criterios de desempeño de los descritos en la Tabla 2, y del descarte de 76 (42\%), clasificados en los subprocesos de control de infecciones hospitalarias, adecuación y ajuste de dosis a nivel hospitalario, salud ocupacional, garantía de la calidad, primeros auxilios, antídotos y vacunas.

Tabla 2. Distribución de los criterios de desempeño del Decreto 3616 del 2005 en los procesos y subprocesos de acuerdo con el Decreto 780 de 2016.

\begin{tabular}{llcc}
\hline \multicolumn{1}{c}{ Proceso } & \multicolumn{1}{c}{ Subproceso específico } & Frecuencia (n) & Porcentaje (\%) \\
\hline & Selección y adquisición & 35 & 19,3 \\
Generales & Información y educación & 26 & 14,4 \\
& Recepción & 15 & 8,3 \\
& Dispensación & 11 & 6,1 \\
& Almacenamiento & 11 & 6,1 \\
Especiales & Control de infecciones hospitalarias & 14 & 7,7 \\
& Demanda insatisfecha & 5 & 2,8 \\
SGC-SO & Adecuación y ajuste de dosis (SDMDU) & 3 & 1,7 \\
Primeros Auxilios & Farmacovigilancia & 2 & 1,1 \\
Antídotos y vacunas & Salud ocupacional & 38 & 21 \\
Total & Garantía de la calidad & 10 & 5,5 \\
SDMDU: & Primeros auxilios & 8 & 4,4 \\
\end{tabular}

SDMDU: sistema de distribución de medicamentos en dosis unitaria. 


\section{b. Revisión de instrumentos de medición de competencias laborales}

En total 993 referencias bibliográficas fueron identificadas con la estrategia de búsqueda, y se seleccionaron 9 artículos para su revisión y análisis. En la Figura 1 se ilustra el proceso de identificación y selección de los artículos. Se encontraron 9 referencias que evaluaron globalmente las competencias laborales del personal farmacéutico, distribuidos en 4 países: 4 en Croacia, 2 en Australia, 2 en Inglaterra y 1 en Serbia. Los hallazgos de las competencias evaluadas en estos estudios se describen en la Tabla 3.

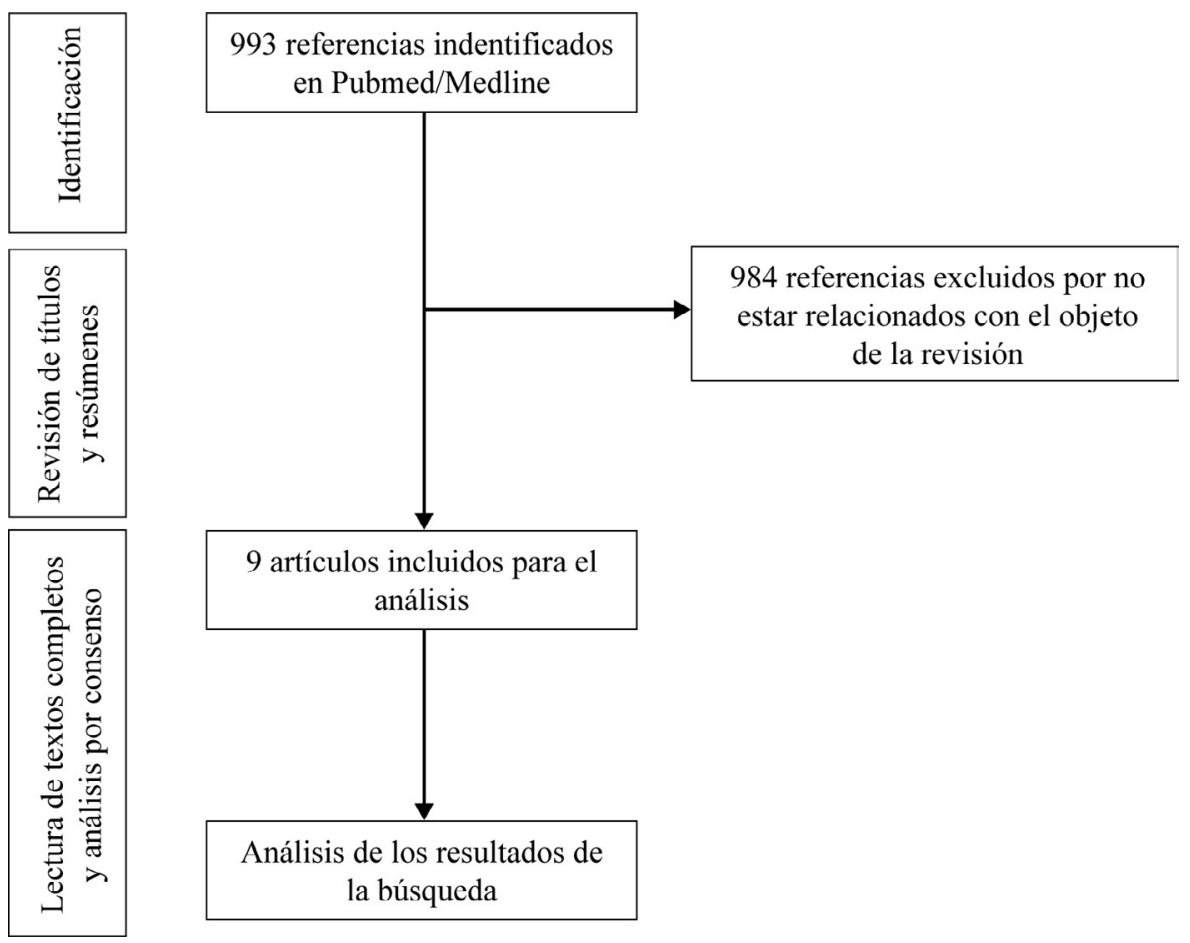

Figura 1. Identificación y selección de artículos para la revisión estructurada.

\section{Construcción del instrumento de medición}

- Diseño de preguntas: las preguntas fueron redactadas en un lenguaje adecuado (se evitaron tecnicismos); sin embargo, debido a la variedad de perfiles laborales que se encuentran en el sistema de salud colombiano para el desempeño en los establecimientos ambulatorios, desde profesionales hasta empíricos, fueron necesarios algunos términos técnicos básicos.

- Evaluación por expertos: el instrumento diseñado fue presentado, revisado y ajustado en diferentes sesiones con la participación de un grupo de profesionales químicos farmacéuticos con experiencia en docencia e investigación en el área farmacéutica asistencial (clínica y comunitaria). Este grupo de profesionales son integrantes activos del grupo de investigación de Promoción y Prevención Farmacéutica de la Universidad de Antioquia (Colombia), y en algunas sesiones habituales del grupo de investigación, se contó con la participación de un médico y de un tecnólogo en regencia de farmacia con experiencia en la dirección técnica de establecimientos farmacéuticos ambulatorios.

- Prueba piloto: posteriormente, el instrumento se sometió a una prueba piloto con 10 personas de diferentes perfiles y funciones, como personal farmacéutico en servicios y establecimientos farmacéuticos ambulatorios, distribuidos así: 2 servicios farmacéuticos ambulatorios con contratos con aseguradoras dentro del sistema de salud, y 5 droguerías y farmacias-droguerías. Luego de la prueba piloto, las observaciones y sugerencias se presentaron, revisaron y ajustaron en una reunión del grupo de químicos farmacéuticos del grupo de investigación. De forma global, solo hubo observaciones relacionadas con la gramática y la redacción de las preguntas. 
Tabla 3. Competencias evaluadas en los instrumentos de medición de los estudios que arrojó la revisión estructurada.

\begin{tabular}{|c|c|c|c|c|c|c|c|c|c|c|}
\hline Dominios & Competencias & $\begin{array}{c}\text { Dzaic } \\
\text { et al, } \\
2018^{(11)}\end{array}$ & $\begin{array}{c}\text { Mucalo } \\
\text { et al, } \\
2^{2016}{ }^{(12)}\end{array}$ & $\begin{array}{c}\text { Stacey } \\
\text { et al, } \\
2^{2015^{(13)}}\end{array}$ & $\begin{array}{c}\text { Svetlana } \\
\text { et al, } \\
\mathbf{2 0 1 4}^{(14)}\end{array}$ & $\begin{array}{c}\text { Mestrovic, } \\
\text { et al, } \\
\mathbf{2 0 1 2}^{(15)}\end{array}$ & $\begin{array}{l}\text { Rutter } \\
\text { et al, } \\
\mathbf{2 0 1 2}^{(16)}\end{array}$ & $\begin{array}{c}\text { Mestrovic, } \\
\text { et al, } \\
\mathbf{2 0 1 1}^{(17)}\end{array}$ & $\begin{array}{c}\text { Coombes } \\
\text { et al, } \\
\mathbf{2 0 1 0}^{(18)}\end{array}$ & $\begin{array}{c}\text { Mills } \\
\text { et al, } \\
\text { 2008 }^{(19)}\end{array}$ \\
\hline \multirow{5}{*}{$\begin{array}{l}\text { Salud pública } \\
\text { (políticas } \\
\text { públicas, } \\
\text { organización y } \\
\text { gestión) }\end{array}$} & $\begin{array}{l}\text { Promoción de la salud } \\
\text { individual y colectiva }\end{array}$ & $\mathrm{x}$ & $\mathrm{x}$ & $\mathrm{x}$ & $\mathrm{x}$ & & $\mathrm{x}$ & & & \\
\hline & $\begin{array}{l}\text { Organización } \\
\text { (estructura organizacional) }\end{array}$ & $\mathrm{x}$ & $\mathrm{x}$ & & & & & & & \\
\hline & Gestión financiera & $\mathrm{x}$ & $\mathrm{x}$ & & & & & & & \\
\hline & Gestión del recurso humano & $\mathrm{x}$ & $\mathrm{x}$ & & & & & & & \\
\hline & Gestión del lugar de trabajo & $\mathrm{x}$ & $\mathrm{x}$ & & & & & & & \\
\hline \multirow{6}{*}{$\begin{array}{l}\text { Vinculadas al } \\
\text { medicamento }\end{array}$} & Evaluación de medicamentos & $\mathrm{x}$ & $\mathrm{x}$ & & & & & & & \\
\hline & Preparación de medicamentos & $\mathrm{x}$ & $\mathrm{x}$ & & & & & & & \\
\hline & Dispensación de medicamentos & $\mathrm{x}$ & & & & & & & & \\
\hline & Selección y adquisición & & $\mathrm{x}$ & $\mathrm{x}$ & & & & & & \\
\hline & $\begin{array}{l}\text { Gestión de la cadena de } \\
\text { suministro }\end{array}$ & $\mathrm{x}$ & $\mathrm{x}$ & $\mathrm{x}$ & & & & & & \\
\hline & Gestión a problemas de calidad & & $\mathrm{x}$ & & & & & & & \\
\hline \multirow{12}{*}{$\begin{array}{l}\text { Vinculadas al } \\
\text { paciente }\end{array}$} & Consejería al paciente & $\mathrm{x}$ & & $\mathrm{x}$ & $\mathrm{x}$ & $\mathrm{x}$ & $\mathrm{x}$ & $\mathrm{x}$ & & \\
\hline & Evaluación de las necesidades & & & $\mathrm{x}$ & $\mathrm{x}$ & $\mathrm{x}$ & $\mathrm{x}$ & $\mathrm{x}$ & & $\mathrm{x}$ \\
\hline & $\begin{array}{l}\text { Evaluación del uso seguro de } \\
\text { los medicamentos }\end{array}$ & $\mathrm{x}$ & & $\mathrm{x}$ & $\mathrm{x}$ & $\mathrm{x}$ & $\mathrm{x}$ & $\mathrm{x}$ & $\mathrm{x}$ & $\mathrm{x}$ \\
\hline & $\begin{array}{l}\text { Provisión del medicamento } \\
\text { (formula médica) }\end{array}$ & & & $\mathrm{x}$ & $\mathrm{x}$ & $\mathrm{x}$ & $\mathrm{x}$ & $\mathrm{x}$ & & \\
\hline & $\begin{array}{l}\text { Información e historia del } \\
\text { paciente }\end{array}$ & & & $\mathrm{x}$ & $\mathrm{x}$ & $\mathrm{x}$ & $\mathrm{x}$ & $\mathrm{x}$ & $\mathrm{x}$ & $\mathrm{x}$ \\
\hline & $\begin{array}{l}\text { Monitorización de la } \\
\text { farmacoterapia }\end{array}$ & & & $\mathrm{x}$ & $\mathrm{x}$ & $\mathrm{x}$ & $\mathrm{x}$ & $\mathrm{x}$ & $\mathrm{x}$ & $\mathrm{x}$ \\
\hline & $\begin{array}{l}\text { Necesidades de información y } \\
\text { educación }\end{array}$ & & & $\mathrm{x}$ & $\mathrm{x}$ & $\mathrm{x}$ & $\mathrm{x}$ & $\mathrm{x}$ & $\mathrm{x}$ & \\
\hline & Evaluación de intervenciones & & & $\mathrm{x}$ & $\mathrm{x}$ & $\mathrm{x}$ & $\mathrm{x}$ & $\mathrm{x}$ & & \\
\hline & $\begin{array}{l}\text { Conocimientos sobre } \\
\text { farmacoterapia }\end{array}$ & & & $\mathrm{x}$ & $\mathrm{x}$ & & & & $\mathrm{x}$ & \\
\hline & $\begin{array}{l}\text { Acceso a información apropiada } \\
\text { (guías y protocolos) }\end{array}$ & & $\mathrm{x}$ & $\mathrm{x}$ & $\mathrm{x}$ & & & & $\mathrm{x}$ & $\mathrm{x}$ \\
\hline & $\begin{array}{l}\text { Documentación de las } \\
\text { actividades }\end{array}$ & & & $\mathrm{x}$ & $\mathrm{x}$ & & $\mathrm{x}$ & & & $\mathrm{x}$ \\
\hline & $\begin{array}{l}\text { Gestión y reporte de eventos } \\
\text { adversos }\end{array}$ & & $\mathrm{x}$ & & & & & & & \\
\hline \multirow{8}{*}{$\begin{array}{l}\text { Profesionales y } \\
\text { personales }\end{array}$} & Habilidades de comunicación & $\mathrm{x}$ & & $\mathrm{x}$ & $\mathrm{x}$ & & & & $\mathrm{x}$ & \\
\hline & Normatividad y practica legal & $\mathrm{x}$ & $\mathrm{x}$ & & & & & & & \\
\hline & Ética y práctica profesional & $\mathrm{x}$ & $\mathrm{x}$ & $\mathrm{x}$ & & & & & & \\
\hline & Calidad de la atención & $\mathrm{x}$ & $\mathrm{x}$ & $\mathrm{x}$ & & & & & & $\mathrm{x}$ \\
\hline & Trabajo en equipo & & & $\mathrm{x}$ & $\mathrm{x}$ & & & & & $\mathrm{x}$ \\
\hline & $\begin{array}{l}\text { Cualidades en la práctica } \\
\text { profesionales }\end{array}$ & & $\mathrm{x}$ & $\mathrm{x}$ & $\mathrm{x}$ & & & & $\mathrm{x}$ & \\
\hline & Evalúa su propio desempeño & & $\mathrm{x}$ & $\mathrm{x}$ & & & & & & \\
\hline & Desarrollo profesional continuo & $\mathrm{x}$ & $\mathrm{x}$ & & & & & & $\mathrm{x}$ & $\mathrm{x}$ \\
\hline
\end{tabular}

\section{Estructura final del instrumento de medición construido}

El instrumento construido está distribuido en los siguientes apartados: 1) Información del personal farmacéutico, y 2) Dominios (funciones): a) suministro de cuidados al paciente, b) personales, c) solución de problemas, y d) gestión y organización. Cada uno de estos subgrupos contiene competencias relacionadas entre sí que conforman cada dominio. Cada una de estas competencias tiene una combinación de criterios de desempeño para ser evaluados, de acuerdo con la valoración de la frecuencia en que se realiza, mediante 
una escala tipo Likert con 4 posibles opciones: 1) Nunca: nunca desempeña o realiza el estándar evaluado, o esta actividad no se encuentra dentro de los procesos del establecimiento farmacéutico; 2) Algunas veces: algunas veces desempeña o realiza el estándar evaluado en sus actividades laborales habituales; 3) Usualmente: usualmente desempeña o realiza el estándar evaluado en sus actividades laborales habituales; 4) Siempre: siempre desempeña o realiza el estándar evaluado en sus actividades laborales habituales. El cuestionario final se describe en la Tabla 4.

Tabla 4. Instrumento de medición construido.

\begin{tabular}{|cc}
\multicolumn{1}{c}{ Ítems } & Nunca Algunas veces Usualmente Siempre \\
\hline Contacto inicial con el paciente &
\end{tabular}

1 ¿Se presenta al paciente/usuario para explicar su función (papel/rol) como personal farmacéutico?

¿Al conversar con el paciente/usuario utiliza términos apropiados (sin

2 términos técnicos o de difícil entendimiento para los pacientes) para obtener información relevante de él y de los medicamentos que utiliza?

¿Cumple los procedimientos establecidos por la normatividad

3 colombiana para la obtención, almacenamiento y tratamiento de los datos del paciente/usuario (habeas data)?

4 ¿Al conversar con el paciente/usuario trata en lo posible de obtener información de los medicamentos que utiliza el paciente?

5 ¿Identifica problemas o dificultades en la adherencia del paciente/ usuario a los medicamentos, y actúa para solucionarlos?

6 ¿Identifica, documenta y actúa en caso de alergias y contraindicaciones absolutas asociadas a los medicamentos?

¿Cuándo identifica problemas relacionados con la utilización de los

7 medicamentos u otros problemas de salud, estos son resueltos o se direcciona el paciente al médico o a un químico farmacéutico?

8 ¿El contacto con el paciente/usuario se da en un clima de cordialidad y respeto?

La prescripción (fórmula médica)

9 ¿Se asegura que la prescripción (fórmula médica) es legal y cumple con las especificaciones de acuerdo con la normatividad?

10 ¿Se asegura que toda la información de la prescripción (fórmula médica) es clara?

11 ¿Se asegura que la prescripción está firmada por un médico u odontólogo?

¿Se asegura que los datos de la prescripción (fórmula médica)

12 concuerdan con la identificación del paciente/usuario y el medicamento a dispensar?

\section{Necesidad de los medicamentos del paciente}

13 ¿Se asegura que los medicamentos dispensados al paciente/usuario son necesarios de acuerdo con los problemas de salud?

¿Se asegura que, si existen errores o discrepancias en los medicamentos

14 del paciente/usuario, estos son documentados, se resuelven o se actúa apropiadamente direccionando el paciente al médico o a un químico farmacéutico?

\section{Conciliación de los medicamentos}

15 ¿Identifica y documenta de forma completa y precisa los otros medicamentos que el paciente/usuario utiliza?

¿Evalúa posibles problemas con los otros medicamentos del paciente,

16 resolviendo o direccionando el paciente al médico o a un químico farmacéutico? 


\section{Suministro de los medicamentos}

¿Se asegura que todos los medicamentos se encuentran almacenados

17 apropiadamente y de manera oportuna, de acuerdo con sus especificaciones de codificación, localización, grupo farmacológico y normas de seguridad?

18 ¿Se hace seguimiento a cualquier problema de suministro y se comunica al responsable del establecimiento?

19 ¿Se asegura que la etiqueta del medicamento dispensado incluya la información requerida y sea apropiada para el paciente?

20 ¿Garantiza un buen proceso de recepción técnica y administrativa de los medicamentos?

\section{Uso de guías y protocolos}

21 ¿Utiliza guías y protocolos como fuente de consulta para brindar información a pacientes/cuidadores?

22 ¿Utiliza un sistema de información de medicamentos como fuente de consulta?

\section{Especificaciones de los medicamentos}

23 ¿Reconoce las indicaciones de los medicamentos prescritos?

¿Reconoce los medicamentos clasificados como de alto riesgo y se

24 tiene en cuenta cualquier precaución o proceso adicional según sea necesario?

25 ¿Se identifican las dosis inadecuadas de los medicamentos y se remite el paciente al médico tratante o a un químico farmacéutico?

26 ¿Se identifican las vías de administración inadecuadas y se remite el paciente al médico tratante o a un químico farmacéutico?

27 ¿Se identifican las frecuencias de uso inadecuadas y se remite el paciente al médico tratante o a un químico farmacéutico?

¿Se asegura que el medicamento dispensado corresponde al principio

28 activo, la dosis, frecuencia y la forma farmacéutica descritas en la prescripción (fórmula médica)?

¿Verifica la calidad del empaque y la información de la etiqueta de los

29 medicamentos al momento de dispensar, principalmente información como las recomendaciones de uso y la fecha de vencimiento?

\section{Interacciones de los medicamentos}

30 ¿Durante el proceso de la dispensación se analiza la posibilidad de interacciones medicamentosas?

\section{Información sobre los medicamentos y educación al paciente}

31 ¿Proporciona consejos sobre estilos de vida saludables (promoción de la salud y prevención de la enfermedad)?

32 ¿En la información suministrada al paciente/usuario se tiene en cuenta sus necesidades individuales?

33 ¿Utiliza alguna estrategia para clasificar los pacientes/usuarios de acuerdo con sus necesidades?

34 ¿Identifica las necesidades de educación para la salud en aquellos pacientes/usuarios que lo requieran?

¿Brinda información clara y completa sobre el uso adecuado de los

35 medicamentos, de acuerdo con la vía de administración, la forma farmacéutica, la frecuencia, el tiempo de uso y el almacenamiento correcto? 


\section{Ítems}

36 ¿Entrega al paciente/usuario información por escrito sobre el uso adecuado de los medicamentos, estilos de vida saludable, etc.?

¿Brinda información sobre los posibles efectos adversos (efectos

37 secundarios) más frecuentes y ofrece posibles recomendaciones para su control y manejo (farmacológicas y no farmacológicas)?

38 ¿Brinda información al paciente/usuario sobre la importancia de la adherencia al tratamiento?

39 ¿Proporciona al paciente/usuario información y orientación sobre los deberes y derechos en salud?

¿La información suministrada al paciente/usuario se encuentra

40 documentada y almacenada en un sistema de información (software, bases de datos)?

¿Usted o en su farmacia/droguería diseña y desarrolla materiales

41 educativos para la promoción de la salud, y prevención de la enfermedad e información sobre el uso adecuado de los medicamentos?

¿Suministra materiales educativos desarrollados por otras personas, universidades o asociaciones para la promoción de la salud, prevención de la enfermedad e información sobre la utilización adecuada de los medicamentos?

\section{Actividades adicionales}

43 ¿Identifica a los pacientes que se automedican y proporciona apoyo según sea necesario?

\section{Identificación de problemas relacionados con los medicamentos}

¿Cuándo identifica problemas relacionados con el uso de los

44 medicamentos o problemas de salud, estos son resueltos o direcciona al paciente al médico o al químico farmacéutico?

\section{Cuidados al paciente}

45 iIdentifica los pacientes que utilizan ayudas para mejorar la adherencia a los medicamentos?

46 ¿Identifica los nuevos pacientes que requieren ayudas para mejorar la adherencia a los medicamentos?

\section{Evaluación de resultados}

47 ¿Realiza la documentación o registro de las intervenciones realizadas a los pacientes/usuarios?

48 ¿Realiza seguimiento y evaluación a las intervenciones realizadas a los pacientes/usuarios para evaluar el aporte de la atención al paciente?

\section{Organización personal}

49 ¿Prioriza la atención al momento de estar en contacto con el paciente/ usuario?

50 ¿Es puntual con el paciente/usuario si hubo compromisos adquiridos previamente?

51 ¿Demuestra iniciativa al momento de estar en contacto con el paciente/ usuario?

52 ¿Usa el tiempo eficientemente al momento de estar en contacto con el paciente/usuario

53 ¿El área de trabajo (mostrador y demás áreas de la Farmacia/Droguería) se conserva ordenado?

¿Presenta una imagen y presentación personal adecuada para la

54 atención a los pacientes/usuario? 
Habilidades de comunicación efectiva

55 ¿Se establece una comunicación clara y precisa con el paciente/cliente?

56 ¿Se establece una comunicación apropiada y de calidad con el paciente/cliente?

57 ¿Se establece una comunicación clara y precisa con otros profesionales de la salud (médico y químicos farmacéuticos)?

58 ¿Se establece una comunicación apropiada y de calidad con otros profesionales de la salud (médico y químicos farmacéuticos)?

59 ¿Se establece una comunicación clara y precisa dentro del equipo de trabajo de la Farmacia/Droguería?

60 ¿Se establece una comunicación apropiada y de calidad dentro del equipo de trabajo de la Farmacia/Droguería?

\section{Equipo de trabajo}

61 ¿Para usted hay respeto y cortesía dentro del equipo de trabajo de la Farmacia/Droguería?

62 ¿Reconoce el valor del resto de personal farmacéutico que labora en la Farmacia/Droguería?

63 ¿Trabaja eficazmente como parte de un equipo de trabajo dentro de la Farmacia/Droguería?

¿Reconoce el valor del otro personal de salud (médicos, nutricionistas,

64 químicos farmacéuticos, psicólogos) que tiene contacto con el paciente?

¿Usa apropiadamente los canales de comunicación y remisión para

65 referir los pacientes a otros profesionales de la salud como médico y químico farmacéutico?

\section{Profesionalismo}

66 ¿Tiene actitud positiva y de servicio al momento de estar en contacto con el paciente/usuario?

67 ¿Guarda confidencialidad de la información del paciente, sus problemas de salud y los medicamentos utilizados?

68 ¿Reconoce sus limitaciones académicas y profesionales con relación a los conocimientos sobre los medicamentos?

69 ¿Se responsabiliza de sus propias acciones hacia la atención y servicios ofrecidos a los pacientes/usuarios?

70 ¿Asume responsabilidades en el cuidado del paciente/usuario?

71 ¿Reflexiona sobre su propio rendimiento académico y profesional?

72 ¿Identifica sus necesidades de aprendizaje?

73 ¿Se actualiza y capacita periódicamente de acuerdo con sus necesidades?

74 ¿Registra sus actividades de aprendizaje?

75 ¿Aplica y evalúa su aprendizaje?

\section{Sobre la información}

76 ¿Accede a información desde recursos apropiados, como sistemas de información y fuentes bibliográficas de validez científica?

77 ¿De la información recopilada resalta los puntos claves?

78 ¿Para su desempeño laboral se mantiene actualizado? 
Revisión, diseño y construcción de un instrumento de medición para evaluar las competencias laborales del personal farmacéutico ambulatorio

\section{Ítems}

Nunca Algunas veces Usualmente Siempre

Conocimiento

¿Reconoce los aspectos biofarmacéuticos, farmacocinéticos y

79 farmacodinámicos de los medicamentos de mayor frecuencia de dispensación?

80 ¿Reconoce los efectos secundarios frecuentes y graves de los medicamentos de mayor rotación o frecuencia de dispensación?

\section{Análisis de la información}

81 ¿Evalúa la información recopilada del paciente/usuario?

¿Identifica los problemas relacionados con la utilización de los

82 medicamentos, a partir de la información suministrada por el paciente/ usuario?

¿Evalúa las opciones de intervención al identificar problemas

83 relacionados con la utilización de los medicamentos en el paciente/ usuario?

84 ¿Toma decisiones al identificar problemas relacionados con la utilización de los medicamentos en el paciente/usuario?

¿Demuestra un enfoque lógico para la resolución de problemas

85 relacionados con la utilización de los medicamentos en el paciente/ usuario?

86 ¿Utiliza alguna estrategia para detectar y clasificar los pacientes/ usuarios de acuerdo con sus necesidades o riesgos?

\section{Proporciona información}

87 ¿Provee información clara y precisa a los pacientes/usuarios?

88 ¿Provee información oportuna a los pacientes/usuarios?

89 ¿Provee información de calidad y relevante a los pacientes/usuarios?

Seguimiento

90 ¿Hace seguimiento a la resolución de los problemas relacionados con la utilización de los medicamentos en el paciente/usuario?

\section{Gestión clínica y Farmacoseguridad}

91 ¿Documenta y reporta los eventos críticos relacionados con la calidad, efectividad y seguridad de los medicamentos?

92 ¿Aplica los principios de seguridad de los medicamentos en la práctica de la dispensación?

¿Las reacciones adversas de los medicamentos son identificadas,

93 registradas y reportadas al programa nacional de farmacovigilancia (DSSA -INVIMA)?

\section{Provisión del servicio}

¿Alerta al director técnico o responsable del establecimiento de

94 posibles mejoras en la calidad de los servicios ofrecidos a los pacientes/ usuarios?

\section{Adquisición}

¿Obtiene los medicamentos mediante un proceso de selección y

95 adquisición de acuerdo con las recomendaciones y sugerencias para realizar estos procesos?

¿Los problemas de abastecimiento de los medicamentos

96 (disponibilidad) se resuelven rápidamente y se toman las medidas correctivas pertinentes?

97 ¿Realiza un control de inventarios para evitar problemas de abastecimiento de los medicamentos? 


\section{Ítems}

Nunca Algunas veces Usualmente Siempre

98 ¿Garantiza que el inventario utilizado o comprado maximiza la rentabilidad?

99 ¿Verifica las existencias de los medicamentos de control especial (en caso tal que dispense estos medicamentos)?

\section{Satisfacción de los servicios ofrecidos}

100 ¿Utiliza algún mecanismo de recepción de PQRS (preguntas, quejas, reclamos y sugerencias) y/o felicitaciones en los pacientes/usuarios?

101 ¿Valora periódicamente la satisfacción del paciente/usuario con relación a los servicios ofrecidos?

\section{Discusión}

Este estudio se fundamentó en describir el proceso de revisión, diseño y construcción de un instrumento de medición para evaluar las competencias laborales del personal farmacéutico ambulatorio en el contexto del sistema de salud colombiano. El instrumento propuesto puede ayudar a otros sistemas de salud de la región a implementar y desarrollar actividades e intervenciones educativas focalizadas en evaluar y mejorar las competencias laborales de este personal de la salud. En este contexto, los resultados de una revisión previa, sobre los programas e intervenciones educativas ofrecidas al personal farmacéutico y los métodos y estrategias utilizadas para evaluar la efectividad de estos programas, encontró que las competencias laborales son uno de los desenlaces más evaluados a nivel mundial. Además, se identificó que la mayoría de este tipo de programas e intervenciones se realiza en países de ingresos medios-altos, y utilizan diferentes marcos de competencias e instrumentos de medición para evaluar el logro de los objetivos, de acuerdo con el contexto de cada país y a las características propias de cada intervención y/o programa desarrollado. Además, se resalta la importancia de este tipo herramientas de medición, debido a que proporciona una estructura para el desarrollo laboral del personal farmacéuticos ${ }^{11}$.

De acuerdo con la estructura del instrumento de medición propuesto, un primer bloque de competencias laborales se estableció bajo el dominio de suministro de cuidados al paciente, el cual, pretende evaluar los comportamientos del personal farmacéutico asociados al contacto inicial con los pacientes. En este caso, el personal farmacéutico describirá si realiza una introducción de sus funciones y de su rol dentro del sistema de salud. Además, valorará su labor, de acuerdo con las actividades y procesos centralizados en la obtención de información del paciente, sus problemas de salud, los medicamentos utilizados y la identificación de interacciones de los medicamentos, así como el suministro de información y educación al paciente, y las fuentes de consulta para ello. Como complemento, se cuestiona por la identificación de problemas relacionados con los medicamentos y la necesidad de actividades adicionales que requiera el paciente $\mathrm{y}$, finalmente, se evalúa los resultados de las atenciones brindadas.

En el segundo dominio, se propone la valoración de las competencias personales. En este bloque se evalúa las competencias propias del personal farmacéutico, enfocadas en el modo como ejecuta sus actividades desde su organización personal, el profesionalismo y las habilidades de comunicación y de trabajo en equipo. En el tercer dominio, descrito como solución de problemas, se valoran las competencias enfocadas al conocimiento y análisis, proporción y seguimiento de la información para el abordaje de los problemas que presente el paciente o sean identificados durante la atención. Finalmente, en el último bloque se evalúa la gestión y organización de las actividades y procesos técnicos, administrativos y asistenciales, como la gestión clínica y la seguridad asociada a la utilización de los medicamentos, la adquisición y selección de los medicamentos, además de la valoración de la provisión y la satisfacción de los servicios ofrecidos.

La evaluación de las competencias laborales constituye una de las actividades relevantes para garantizar el óptimo desempeño laboral. Por ello, se debe dedicar especial atención al diseño y la creación de instrumentos útiles que aporten información fiable sobre el desempeño del personal farmacéutico, a partir de las competencias propias necesarias para un adecuado funcionamiento de los Establecimientos Farmacéuticos ambulatorios. El instrumento de evaluación propuesto, además de ser utilizado para la medición de las competencias laborales, permite la mejora continua de los procesos y servicios establecidos en la normatividad y por las organizaciones y asociaciones a nivel internacional, que pueden conducir a un plan de acciones, orientado 
a mejorar la calidad de la atención en salud. El instrumento construido favorecerá la integración de procesos investigativos, formativos y laborales.

Las droguerías y farmacias-droguerías pueden jugar un papel importante en la mejora de la farmacoterapia y los resultados en la salud de los pacientes, al promover el uso racional de los medicamentos y reducir los costos de salud ${ }^{9,12}$. De acuerdo con la normatividad colombiana, existen dos características específicas que diferencian una droguería de una farmacia-droguería, la primera es que en una farmacia-droguería se pueden realizar preparaciones magistrales y la segunda es que la dirección técnica de estás, estarán a cargo por un Químico Farmacéutico o, en su defecto, cuando las preparaciones magistrales consisten en preparaciones no estériles, podrá ser un Tecnólogo en Regencia de Farmacia. Por tanto, las diferencias entre estos dos establecimientos están determinadas principalmente por la realización o no de este proceso, y en caso tal, será de obligatoriedad la dirección técnica por parte de una persona con formación universitaria (profesional o tecnológica).

Por tanto, las funciones para el desempeño de los procesos y los servicios técnicos y asistenciales, a nivel ambulatorio de estos establecimientos, no están determinadas por las diferencias o particularidades en los perfiles laborales del personal farmacéutico. Por ello, la importancia de aumentar, mejorar, evaluar y sostener en el tiempo, un programa de educación continua y/o de desarrollo profesional para mejorar las competencias laborales ${ }^{11}$. En este sentido, la disponibilidad de la herramienta generada con este trabajo cumple con aspectos y requisitos metodológicos para evaluar y seguir las competencias laborales del personal farmacéutico en el tiempo. En el caso específico de Colombia, un estudio reciente, orientado a evaluar las habilidades y actitudes del personal farmacéutico ambulatorio, en la dispensación de tramadol en las droguerías y farmaciasdroguerías, evidenció la necesidad de generar y desarrollar estrategias para evaluar las competencias laborales requeridas para contribuir a la calidad del uso de los medicamentos, minimizar los errores de medicación y ayudar a los pacientes ambulatorios a administrar mejor sus medicamentos ${ }^{13}$.

\section{Conclusión}

Se revisan y analizan las directrices y recomendaciones internacionales, además, la normatividad colombiana para identificar las competencias laborales que requiere el personal farmacéutico para el correcto funcionamiento de los Establecimientos Farmacéuticos ambulatorios $\mathrm{y}$, con este soporte, se construye un instrumento de medición de dichas competencias. El instrumento construido con los dominios (funciones) y criterios de desempeño propuestos puede ser utilizado para valorar y seguir las competencias laborales en este personal de la salud; sin embargo, se requiere de estudios diseñados para valorar la funcionalidad del instrumento $\mathrm{y}$, a su vez, contribuyan a su validación.

\section{Agradecimientos}

A la UDEA y a la Facultad de Ciencias Farmacéuticas y Alimentarias de la Universidad de Antioquia.

\section{Financiación}

El grupo Promoción y Prevención Farmacéutica recibió financiación de la convocatoria de sostenibilidad 20182019 del Comité para el Desarrollo de la Investigación de la Universidad de Antioquia.

\section{Conflicto de interés}

Los autores declaran no tener ningún conflicto de intereses en el trabajo realizado.

\section{Referencias}

1. Organización Panamericana de la Salud (OPS). Conferencia Panamericana de Educación Farmacéutica (CPEF). Propuesta de Plan Básico de Educación Farmacéutica y Competencias del Farmacéutico para la práctica profesional Conferencia Panamericana de Educación Farmacéutica (CPEF).

2. Organización Mundial de la Salud (OMS), Federación Internacional Farmacéutica (FIP). Competencias del farmacéutico para desarrollar los servicios farmacéuticos basados en Atención Primaria de Salud y las Buenas Prácticas en Farmacia. Grupo Técnico para el desarrollo de competencias para los Servicios Farmacéuticos (GT-CSF. 2012.

3. República de Colombia. Ministerio de Salud y Protección Social. Decreto 3616. Por medio del cual se establecen las denominaciones de los auxiliares en las áreas de la salud, se adoptan sus perfiles ocupacionales y de formación, los requisitos básicos de calidad de sus programas y se dictan otras disposiciones. 2005. 
4. República de Colombia. Ministerio de Salud y Protección Social. Decreto 780. Por medio del cual se expide el Decreto Único Reglamentario del Sector Salud y Protección Social. 2016.

5. República de Colombia. Ministerio de Salud y Protección Social. Resolución 1963. Por la cual se definen las características específicas de calidad para la oferta y desarrollo del programa académico de Tecnología en Regencia de Farmacia. 2006.

6. República de Colombia. Ministerio de Salud y Protección Social. Resolución 1964. Por la cual se definen las características específicas de calidad para la oferta y desarrollo del programa académico del nivel de formación profesional en Farmacia. 2006.

7. Arroyave $\mathrm{O}$, Álvarez W, Ameriles $\mathrm{P}$, Vásquez M, Cardona B. Pertinencia laboral y social del progrma de Tecnología en Regencia de Farmacia en la región de Urabá. Rev Univ Ind Santander Salud. 2016; 48(4): 526-534. doi: http://dx.doi. org/10.18273/revsal.v48n4-2016011

8. Ceballos M, Giraldo J, Giraldo J, Marín V, Amariles P. Caracterización de aspectos relacionados con la utilización de los medicamentos fiscalizados en droguerías y farmacias-droguerías de Medellín y el Área Metropolitana. Rev Univ Ind Santander Salud. 2018; 50(1): 27-36. doi: 10.18273/revsal.v50n12018003

9. Organización Mundial de la Salud (OMS), Federación Internacional Farmacéutica (FIP). Directrices Conjuntas FIP/OMS sobre Buenas Prácticas en Farmacia: Estándares para la calidad de los servicios farmacéuticos. 2011.

10. Federation International Pharmaceutical (FIP). Pharmacy Education Taskforce A Global Competency Framework. 2012.

11. Držaić M, Kummer I, Mucalo I, Bruno A, Ortner Hadžiabdić M. Identifying self-assessed competencies and areas for improvement within community pharmacist-preceptors support during pre-registration training. BMC Med Educ. 2018; 18(1): 303 .

12. Mucalo I, Hadžiabdić MO, Govorčinović T, Šarić M, Bruno A, Bates I. The development of the Croatian competency framework for pharmacists. Am J Pharm Educ. 2016; 80(8): 134.

13. Stacey SR, Coombes I, Cardiff L, Wainwright C, Whitfield KM. Using the General Level Framework to guide training and development needs of pharmacists working in pediatrics. J Pharm Pract Res. 2015;45(3): 322-330.
14. Svetlana S, Ivana T, Tatjana C, Duskana K, Ian B. Evaluation of competences at the community pharmacy settings. Indian J Pharm Educ Res. 2014; 48(4): 22-30.

15. Meštrović A, Staničić Z, Hadžiabdić MO, Mucalo I, Bates I, Duggan C, et al. Individualized education and competency development of Croatian community pharmacists using the general level framework. Am J Pharm Educ. 2012; 76(2): 23. doi: 10.5688/ajpe76223

16. Rutter V, Wong C, Coombes I, Cardiff L, Duggan C, Yee M-L, et al. Use of a general level framework to facilitate performance improvement in hospital pharmacists in Singapore. Am J Pharm Educ. 2012; 76(6): 107. doi: 10.5688/ajpe766107

17. Meštrović A, Staničić Ž, Hadžiabdić MO, Mucalo I, Bates I, Duggan C, et al. Evaluation of Croatian Community Pharmacists' patient care competencies using the general level framework. Am J Pharm Educ. 2011; 75(2): 36. doi: 10.5688/ajpe 75236

18. Coombes I, Avent M, Cardiff L, Bettenay K, Coombes J, Whitfield $\mathrm{K}$, et al. Improvement in Pharmacist's Performance Facilitated by an Adapted Competency-Based General Level Framework. J Pharm Pract Res. 2010; 40(2): 111-118.

19. Mills E, Farmer D, Bates I, Davies G, Webb DG. The general level framework: use in primary care and community pharmacy to support professional development. Int J Pharm Pract. 2008; 16(5): 325 331. doi: https://doi.org/10.1211/ijpp.16.5.0008 